\title{
Quantum critical point in the spin glass-antiferromagnetism competition for fermionic Ising Models
}

\author{
F. M. Zimmer and S. G. Magalhães* \\ Departamento de Física, Universidade Federal de Santa Maria \\ 97111-900 Santa Maria, RS, Brazil.
}

\begin{abstract}
The competition between spin glass $(S G)$ and antiferromagnetic order $(A F)$ is analyzed in two sublattice fermionic Ising models in the presence of a transverse $\Gamma$ and a parallel $H$ magnetic fields. The exchange interaction follows a Gaussian probability distribution with mean $-4 J_{0} / N$ and standard deviation $J \sqrt{32 / N}$, but only spins in different sublattices can interact. The problem is formulated in a path integral formalism, where the spin operators have been expressed as bilinear combinations of Grassmann fields. The results of two fermionic models are compared. In the first one, the diagonal $S^{z}$ operator has four states, where two eigenvalues vanish ( $4 \mathrm{~S}$ model), which are suppressed by a restriction in the two states $2 \mathrm{~S}$ model. The replica symmetry ansatz and the static approximation have been used to obtain the free energy. The results are showing in phase diagrams $T / J$ ( $T$ is the temperature) versus $J_{0} / J, \Gamma / J$, and $H / J$. When $\Gamma$ is increased, $T_{f}$ (transition temperature to a nonergodic phase) reduces and the Neel temperature decreases towards a quantum critical point. The field $H$ always destroys $A F$; however, within a certain range, it favors the frustration. Therefore, the presence of both fields, $\Gamma$ and $H$, produces effects that are in competition. The critical temperatures are lower for the $4 \mathrm{~S}$ model and it is less sensitive to the magnetic couplings than the $2 \mathrm{~S}$ model.
\end{abstract}

\section{Introduction}

There are now several examples of competition between antiferromagnetism (AF) and spin glass (SG) in strongly correlated systems as, for instance, heavy fermions and high $T_{c}$ superconductors [1] 2]. In some of these systems, there is also a new physics involved with the presence of a quantum critical point (QCP) and deviation of the Fermi liquid behavior, so called Non-Fermi liquid (NFL) behavior. This raises the necessity of obtaining a solid framework to describe those systems where the fermions are operative to give origin to physical processes, such as the Kondo effect, in connection with the presence of $\mathrm{AF}$ and frustration driven by disorder, particularly, at low temperatures where quantum effects become important.

The complexities of such description can be estimated by the controversies [3] involved in the quantum Ising SG which has been investigated by several techniques 4 . For instance, one of the most interesting open issues is whether the quantum tunneling between the local degenerated minimum of the free energy is able to stabilize the replica symmetric solution of the problem. Recently, the fermionic Ising SG in the presence of a transverse magnetic field $\Gamma$ has been studied using the functional integral formalism [5]. The spins have been represented by bilinear combinations of the fermion operators. This fermionic problem has been presented in two versions. In the first one, the fermionic spin operators have maintained their four natural eigenvalues, where two of them are non-magnetic. In the second one, it has been retained only the magnetic ones due to an imposed constraint to garantee complete equivalence between the spin and the fermionic problem 6 . The free energy has been obtained in both versions within the replica symmetric theory and the static

*E-mail address: ggarcia@ccne.ufsm.br 
approximation. It has been found that the freezing temperature $T_{f}(\Gamma)$ decreases to a QCP when $\Gamma$ enhances in a second order type transition. In that formulation, the replica symmetric solution is unstable in the entire SG region. The fermionic formulation is also a natural tool to study the interplay between frustration driven by disorder and, for instance, Kondo effect at low temperature when quantum tunneling is important. Actually, quite recently, the SG problem with transverse field has been studied successfully in the disordered Kondo lattice with this technique [7.

The purpose of the present work is to investigate the SG/AF competition in the context of the fermionic representation for the Ising spins variables when a magnetic field is applied with two components: one parallel $(H)$ and other transverse $(\Gamma)$ to the $z$ direction. The component $\Gamma$ introduces a spin flipping mechanism in the problem which can lead the phase boundaries to a QCP [5]. The model used here is the fermionic version of the Korenblit-Shender (KS) model 8] introduced to study SG/AF competition with classical Ising spins. In the KS model, there are two sublattices, but only spins in distinct sublattices are allowed to interact with a random Gaussian coupling $J_{i j}$. The presence of the magnetic field $H$ breaks the symmetry between the sublattices. As consequence, it can introduce unusual effects as it has been shown in the mean field theory with replica symmetry for the classical interlattice frustrated model 8 . For instance, the field $H$ can favor frustration within a certain range. This is in contrast with the well known result from the Sherrington-Kirkpatrick model (SK) 9] where the freezing temperature $T_{f}$, associated with the Almeida-Thouless instability, decreases monotonically with the field $H$. In fact, the dependence of the random internal field with $H$ in the KS model could explain its odd behavior [8]. The internal field applied $h_{p}$ in a particular sublattice $(p=a, b)$ depends on the magnetization $m_{p^{\prime}}$ and the SG order parameter $q_{p^{\prime}}\left(p^{\prime} \neq p\right)$. Due to the break of the symmetry between the two sublattices, when $H$ is increased in a particular range, one may have a nonmonotonic behavior in the order parameters $m_{p^{\prime}}, q_{p^{\prime}}$ and, consequently, in the $h_{p}$. Therefore, $T_{f}$ can be enhanced and the non-trivial ergodicity breaking region (SG) is enlarged.

This leads several issues for the SG/AF competition in the fermionic representation of the KS model, when both field components $(H$ and $\Gamma)$ are applied. Is the scenario described previously for the classical KS model preserved or not even for $\Gamma=0$ case? The answer is not obvious because the fermionic representation of the spin operators introduces an important difference as compared with its classical counterpart. The replica diagonal spin glass order parameter for the two sublattices is not constrained to the unity. It must be solved together with the other order parameters, the replica off-diagonal SG order parameter and the magnetization for the two sublattices. As consequence, it would introduce a new component in the random internal field. If the previous question is answered positively, is there any range of $H$ which can favor frustration when $\Gamma \neq 0$ as in the classical KS? The presence of $H$, by a mechanism similar to the its classical counterpart, could enhance $T_{f}$ while the $\Gamma$ component tends to suppress frustration leading the $T_{f}$ to a QCP [5]. Therefore, the fields $H$ and $\Gamma$ enforce two competing mechanisms in the problem which could deeply affect the SG/AF phase boundaries.

The quantum mechanical partition function of the problem has been obtained following the approach introduced in reference [5]. Therefore, the functional integral approximation is used to deal with non-commutativity of the spin operators which are represented by bilinear combinations of Grassmann variables. One important aspect of the fermionic representation of spins $S_{i}^{z}$ is that (see Ref. [5]) it has four eingenvalues per site, where two of them are non-magnetic. For the rest of the paper, this representation is named $4 \mathrm{~S}$ model. To recover the usual spin representation, a constraint is introduced in order to maintain only the magnetic eigenvalues. This version of the problem is named $2 \mathrm{~S}$ model. The disorder in the problem is treated using the replica trick where the order parameters are obtained within the replica symmetric ansatz [9. It should be noticed that the possible occupation of non-magnetic states can produce differences concerning the phase boundaries between the two models. In fact, for the one lattice fermionic SG with transverse field [5], $\left(T_{f}\right)_{2 S}>\left(T_{f}\right)_{4 S}$ for $\Gamma<<\Gamma_{c}$ where $\Gamma_{c}$ is the value of the field at the QCP. This also arises the question how these representations $2 \mathrm{~S}$ and $4 \mathrm{~S}$ respond (in terms of the phase boundaries) when $H$ and $\Gamma$ are turned on. In order to allow us a better comparision between the two representations of the problem, the $4 \mathrm{~S}$ model is kept in the half-filling. 
In the present approach, the time dependence of the spin-spin correlation functions has not been considered (static approximation) 10. The main argument to justify the use of this approximation (see Ref. [5] 11), even at low temperature, is that our goal is to obtain the phase boundaries in the SG/AF competition in the spin fermionic representation and how these boundaries are affected by the presence of $H$ and $\Gamma$. As it can be seen elsewhere [7, 12, that approximation is quite reasonable if the intention is not to reveal the complex nature of phases at very low temperature, but mainly to produce phase boundaries which can mimic the experimental ones.

It is hoped that the results presented here can be a first step to provide a framework which can allow us to study the phase boundaries SG/AF competition present in several problems of many interacting fermions, such as the heavy fermion system $\mathrm{Ce}_{2} A u_{1-x} \mathrm{Co}_{x} S i_{3}$ [13] where disorder, competing RKKY interaction and the Kondo effect have produced a SG alike state and an AF phase where the Neel temperature seems to decrease towards a QCP with no trace of NFL behavior.

This paper has the following structure. In section II, the model is introduced, the saddle point free energy and the corresponding order parameters are obtained. In section III, the numerical solutions of the order parameters allow us to construct phase diagrams to show the AF/SG competition. Particularly interesting, for the purposes of the present work, are the phase diagrams in the space temperature versus the components of the magnetic fields $H$ and $\Gamma$. It is also shown the behavior of the susceptibility. In the last section, the discussion of the results previously shown and the final remarks are presented.

\section{Model}

The model considered here is a fermionic Ising model 14 represented in two sublattices $a$ and $b$ where there are two magnetic fields applied: $\Gamma$ and $H$ transverse and parallel to the Ising spins, respectively. One important point is that only spins located in distinct sublattices can interact like in the KS model [8]. Thus,

$$
\hat{H}=-\sum_{i_{a} j_{b}} J_{i_{a} j_{b}} \hat{S}_{i_{a}}^{z} \hat{S}_{j_{b}}^{z}-2 \sum_{i_{a}}\left(\Gamma \hat{S}_{i_{a}}^{x}+H \hat{S}_{i_{a}}^{z}\right)-2 \sum_{j_{b}}\left(\Gamma \hat{S}_{j_{b}}^{x}+H \hat{S}_{j_{b}}^{z}\right)
$$

where the sums are run over the $N$ sites of each sublattice ( $a$ or $b$ ). The coupling $J_{i_{a} j_{b}}$ is assumed to be a random variable with a Gaussian distribution given by:

$$
P\left(J_{i_{a} j_{b}}\right)=\sqrt{\frac{N}{64 \pi J^{2}}} \exp \left[-\frac{\left(J_{i_{a} j_{b}}+4 J_{0} / N\right)^{2}}{64 J^{2}} N\right] .
$$

The spin operators in Eq. (11) are defined as [5]:

$$
\hat{S}_{i_{p}}^{z}=\frac{1}{2}\left[\hat{n}_{i_{p} \uparrow}-\hat{n}_{i_{p} \downarrow}\right], \quad \hat{S}_{i_{p}}^{x}=\frac{1}{2}\left[c_{i_{p} \uparrow}^{\dagger} c_{i_{p} \downarrow}+c_{i_{p} \downarrow}^{\dagger} c_{i_{p} \uparrow}\right]
$$

where $\hat{n}_{i_{p} \sigma}=c_{i_{p} \sigma}^{\dagger} c_{i_{p} \sigma}$ is the number operator, $c_{i_{p} \sigma}^{\dagger}\left(c_{i_{p} \sigma}\right)$ are fermions creation (destruction) operators, with $\sigma=\uparrow$ or $\downarrow$ indicating the spin projections, and the sub-index $p=a$ or $b$ represents the sublattice.

In Eq. (3), the spins have been written as bilinear combination of fermion operators which act on a space with four states per site $(|00\rangle,|\uparrow 0\rangle,|0 \downarrow\rangle,|\uparrow \downarrow\rangle)$. Therefore, $\hat{S}_{i_{p}}^{z}$ has four eigenvalues: $\pm 1 / 2$ (when there is one fermion in the site $i_{p}(\sigma=\uparrow$ or $\downarrow)$ ) and two when the site $i_{p}$ is unoccupied or double occupied $(\sigma=\uparrow$ and $\downarrow)$. In the present work, two formulations are considered: one unrestrained the number of states of the $S_{i_{p}}^{z}(4 \mathrm{~S}$ model), but it considers the average occupation of one fermion per site, another restrained the occupation number to $\hat{n}_{i_{p} \uparrow}+\hat{n}_{i_{p} \downarrow}=1$ (2S model). This last representation allow us to study the problem avoiding the presence of unoccupied and double occupied states [5].

The partition function is given in the Lagrangian path integral formalism where the spin operators are represented as anticommuting Grassmann fields $\left(\phi, \phi^{*}\right)$. The partition function for the 
$2 \mathrm{~S}$ model must consider only states that have one fermion per site. This restriction is obtained by using the Kronecker $\delta$ function $\left(\delta\left(\hat{n}_{i_{p} \uparrow}+\hat{n}_{i_{p} \downarrow}-1\right)=\frac{1}{2 \pi} \int_{0}^{2 \pi} d x_{i_{p}} e^{i x_{i_{p}}\left(\hat{n}_{i_{p} \uparrow}+\hat{n}_{i_{p} \downarrow}-1\right)}\right)$. Therefore, the partition function for both models can be represented in a compact form as [5]:

$$
Z\{\mu\}=\prod_{p=a, b} \prod_{i_{p}} \frac{1}{2 \pi} \int_{0}^{2 \pi} d x_{i_{p}} e^{-\mu_{i_{p}}} \int D\left(\phi^{*} \phi\right) \exp (A\{\mu\})
$$

where $\mu_{i_{p}}=0$ for the $4 \mathrm{~S}$ model, which corresponds to the half-filling situation, or $\mu_{i_{p}}=i x_{i_{p}}$ for the $2 \mathrm{~S}$ model. The action $A\{\mu\}$ can be Fourier transformed in time. Thus, we have:

$$
A\{\mu\}=A_{M}^{a}+A_{M}^{b}+A_{S G}
$$

with

$$
\begin{gathered}
A_{M}^{p}=\sum_{i_{p}} \sum_{\omega} \underline{\phi}_{i_{p}}^{\dagger}(\omega)\left[i \omega+\mu_{i_{p}}+\beta H \underline{\sigma}^{z}+\beta \Gamma \underline{\sigma}^{x}\right] \underline{\phi}_{i_{p}}(\omega), \\
A_{S G}=\sum_{\Omega} \sum_{i_{a} j_{b}} \beta J_{i_{a} j_{b}} S_{i_{a}}(\Omega) S_{j_{b}}(-\Omega), \\
S_{i_{p}}(\Omega)=\sum_{\omega} \underline{\phi}_{i_{p}}^{\dagger}(\omega+\Omega) \underline{\sigma}^{z} \underline{\phi}_{i_{p}}(\omega),
\end{gathered}
$$

$\beta$ the inverse temperature and the matrices in Eqs. (6] 8) are defined as:

$$
\underline{\phi}_{i_{p}}(\omega)=\left[\begin{array}{c}
\phi_{i_{p} \uparrow}(\omega) \\
\phi_{i_{p} \downarrow}(\omega)
\end{array}\right] ; \underline{\sigma}^{x}=\left(\begin{array}{cc}
0 & 1 \\
1 & 0
\end{array}\right) ; \quad \underline{\sigma}^{z}=\left(\begin{array}{cc}
1 & 0 \\
0 & -1
\end{array}\right) ;
$$

with the Matsubara's frequencies $\omega=(2 m+1) \pi$ and $\Omega=2 m \pi(m=0, \pm 1, \cdots)$. In this work, the problem is analyzed in the static approximation, which considers only the term when $\Omega=0$ in Eq. (7) $5,10,14$.

The free energy per site is obtained by using the replica method: $\beta F=-\ln Z=-\lim _{n \rightarrow 0}$ $1 /(n N)(Z(n)-1)$, where $Z(n) \equiv\left\langle Z^{n}\right\rangle_{J_{i_{a} j_{b}}}$ is the configurational averaged replicated partition function. The average over $P\left(J_{i_{a} j_{b}}\right)$ can be performed using the Gaussian distribution given in Eq. (2). Thus:

$$
\begin{aligned}
Z(n) & =\prod_{p=a, b} \prod_{i_{p}} \prod_{\alpha=1}^{n} \frac{1}{2 \pi} \int_{0}^{2 \pi} d x_{i_{p}}^{\alpha} e^{-\mu_{i_{p}}^{\alpha}} \int D\left(\phi_{\alpha}^{*}, \phi_{\alpha}\right) \exp \left\{\sum _ { \alpha = 1 } ^ { n } \left(A_{M}^{a, \alpha}\right.\right. \\
& \left.\left.+A_{M}^{b, \alpha}\right)+\sum_{i_{a} j_{b}}\left[\frac{16 \beta^{2} J^{2}}{N}\left(\sum_{\alpha=1}^{n} S_{i_{a}}^{\alpha} S_{j_{b}}^{\alpha}\right)^{2}-\frac{4 \beta J_{0}}{N} \sum_{\alpha=1}^{n} S_{i_{a}}^{\alpha} S_{j_{b}}^{\alpha}\right]\right\}
\end{aligned}
$$

where $\alpha$ denotes the replica index and $S_{i_{p}}^{\alpha} \equiv S_{i_{p}}^{\alpha}(0)$. Eq. (10) can be rearranged reviewing the sums over different sublattices by square sums over the same sublattice [8]. Then, these quadratic terms are linearized by using the Hubbard-Stratonovich transformation. This transformation inserts auxiliary fields $\left\{M_{3}^{\alpha}, M_{p}^{\alpha}, Q_{3}^{\alpha \gamma}\right.$, and $\left.Q_{p}^{\alpha \gamma}\right\}$ on the partition function, therefore:

$$
\begin{aligned}
Z(n)= & \int d U \exp \left\{-N\left[\frac{\beta J_{0}}{2} \sum_{\alpha}\left(\left(M_{3}^{\alpha}\right)^{2}+\sum_{p=a, b}\left(M_{p}^{\alpha}\right)^{2}\right)\right.\right. \\
& \left.\left.+\frac{\beta^{2} J^{2}}{2} \sum_{\alpha \gamma}\left(\left(Q_{3}^{\alpha \gamma}\right)^{2}+\sum_{p=a, b}\left(Q_{p}^{\alpha \gamma}\right)^{2}\right)-\frac{1}{N} \ln \Theta_{\alpha \gamma}\{\mu\}\right]\right\}
\end{aligned}
$$


where $\int d U=\int_{-\infty}^{\infty} \prod_{r=a, b, 3} \prod_{\alpha} d M_{r}^{\alpha} \int_{-\infty}^{\infty} \prod_{r=a, b, 3} \prod_{\alpha \gamma} d Q_{r}^{\alpha \gamma}$, and the functional part is expressed as:

$$
\begin{aligned}
\Theta_{\alpha \gamma}\{\mu\} & =\prod_{p=a, b} \prod_{i_{p}, \alpha} \frac{1}{2 \pi} \int_{0}^{2 \pi} d x_{i_{p}}^{\alpha} e^{-\mu_{i_{p}}^{\alpha}} \int D\left(\phi_{\alpha}^{*}, \phi_{\alpha}\right) \\
& \exp \left\{\sum _ { p = a , b } \left[\sum_{\alpha} A_{M}^{p, \alpha}+2 \beta J_{0} \sum_{\alpha, i_{p}}\left(i M_{3}^{\alpha}+M_{p}^{\alpha}\right) S_{i_{p}}^{\alpha}\right.\right. \\
& \left.\left.+4 \beta^{2} J^{2} \sum_{\alpha \gamma, i_{p}}\left(Q_{3}^{\alpha \gamma}+i Q_{p}^{\alpha \gamma}\right) S_{i_{p}}^{\alpha} S_{i_{p}}^{\gamma}\right]\right\} .
\end{aligned}
$$

In the thermodynamic limit, the set of integrals in $\int d U$ can be performed exactly by the steepest descent method, where the auxiliary fields are given by the saddle point solutions:

$$
\begin{aligned}
& M_{3}^{\alpha}=\frac{i 2}{N}\left\langle\sum_{p=a, b} \sum_{i_{p}} S_{i_{p}}^{\alpha}\right\rangle=2 i m_{3}^{\alpha} ; \quad Q_{3}^{\alpha \gamma}=\frac{4}{N}\left\langle\sum_{p=a, b} \sum_{i_{p}} S_{i_{p}}^{\alpha} S_{i_{p}}^{\gamma}\right\rangle=2 q_{3}^{\alpha \gamma} ; \\
& M_{p}^{\alpha}=\frac{2}{N}\left\langle\sum_{i_{p}} S_{i_{p}}^{\alpha}\right\rangle=m_{p}^{\alpha} ; \quad Q_{p}^{\alpha \gamma}=\frac{i 4}{N}\left\langle\sum_{i_{p}} S_{i_{p}}^{\alpha} S_{i_{p}}^{\gamma}\right\rangle=i q_{p}^{\alpha \gamma} ; p=a, b
\end{aligned}
$$

where $\langle\ldots\rangle$ denotes the average taken with respect to Eq. (12). These saddle point equations can be used to rewrite Eq. (11) as:

$$
Z(n)=\int d U \exp \left\{-N\left[\beta^{2} J^{2} \sum_{\alpha \gamma} q_{a}^{\alpha \gamma} q_{b}^{\alpha \gamma}-\beta J_{0} \sum_{\alpha} m_{a}^{\alpha} m_{b}^{\alpha}-\sum_{p} \ln \Theta_{\alpha \gamma}^{p}\{\mu\}\right]\right\}
$$

where:

$$
\Theta_{\alpha \gamma}^{p}\{\mu\}=\prod_{\alpha} \frac{1}{2 \pi} \int_{0}^{2 \pi} d x_{p}^{\alpha} e^{-\mu_{p}^{\alpha}} \int D\left[\phi_{p}^{*} \phi_{p}\right] \exp \left[H_{p}^{e f f}\right]
$$

with

$$
H_{p}^{e f f}=\sum_{\alpha}\left[A_{M, p}^{\alpha}-2 \beta J_{0} m_{p^{\prime}}^{\alpha} S_{p}^{\alpha}\right]+4 \beta^{2} J^{2} \sum_{\alpha \gamma} q_{p^{\prime}}^{\alpha \gamma} S_{p}^{\alpha} S_{p}^{\gamma},
$$

and $p=a\left(p^{\prime}=b\right)$ or $p=b\left(p^{\prime}=a\right)$.

At this stage, it is assumed the replica symmetric "ansatz" that considers the set $q_{p}^{\alpha \gamma}=q_{p}$ for all $\alpha \neq \gamma, q_{p}^{\alpha \alpha}=q_{p}+\bar{\chi}_{p}$, and $m_{p}^{\alpha}=m_{p}$ for all $\alpha$. The physical quantity $\bar{\chi}_{p}=\chi_{p}^{*} / \beta$ where $\chi_{p}^{*}$ is the magnetic susceptibility when $J_{0}=0$. The sums over $\alpha$ in Eq. (17) produce quadratic terms again, which can be linearized introducing new auxiliary fields in the expression (16), therefore:

$$
\Theta_{\alpha \gamma}^{p}\{\mu\}=\int_{-\infty}^{\infty} D z_{p}\left[\int_{-\infty}^{\infty} D \xi_{p} \frac{1}{2 \pi} \int_{0}^{2 \pi} d x_{p} e^{-\mu_{p}} \Lambda_{e f f}^{p}\right]^{n}
$$

where $D y_{p}=d y_{p} \exp \left(-y_{p}^{2} / 2\right) / \sqrt{2 \pi}$, and

$$
\Lambda_{e f f}^{p}=\int D\left[\phi_{p}^{*} \phi_{p}\right] \exp \left[\sum_{\omega} \underline{\phi}_{p}^{\dagger}(\omega) \underline{G}_{p}^{-1}(\omega) \underline{\phi}_{p}(\omega)\right] .
$$

The matrix $\underline{G}_{p}^{-1}(\omega)$ is given by:

$$
\underline{G}_{p}^{-1}(\omega)=\left(\begin{array}{cc}
i \omega+\mu_{p}+\beta h_{p} & \beta \Gamma \\
\beta \Gamma & i \omega+\mu_{p}-\beta h_{p}
\end{array}\right)
$$

where

$$
h_{p}=\left(H-J_{0} m_{p^{\prime}}+J \sqrt{2 q_{p^{\prime}}} z_{p}+J \sqrt{2 \bar{\chi}_{p^{\prime}}} \xi_{p}\right)
$$


with $p^{\prime} \neq p$. Therefore, the internal field $h_{p}$ applied in a particular sublattice depends entirely on the order parameters of the other sublattice [8].

The functional integral over the Grassmann variables in Eq. (19) can be calculated (see Ref. [15]), thus, $\ln \Lambda_{\text {eff }}^{p}=\sum_{\omega} \ln \left(\operatorname{det} \underline{G}_{p}^{-1}(\omega)\right)$. In the same equation, the sum over the Matsubara's frequencies can be performed like references [5, 14] to give the following expression:

$$
\Lambda_{e f f}^{p}=2 e^{\mu_{p}}\left[\cosh \mu_{p}+\cosh \left(\beta \sqrt{\Delta_{p}}\right)\right]
$$

where $\Delta_{p}=h_{p}^{2}+\Gamma^{2}$.

Now, the restriction condition over the number of states can be used for both models. For the $4 \mathrm{~S}$ model, the average occupation per site is one. It is obtained putting $\mu_{p}=0$, therefore the integral over $x_{p}$ is equal to the unity. For the $2 \mathrm{~S}$ model $\left(\mu_{p}=i x_{p}\right)$, which corresponds to the spin formulation where there are only two states per site $(|\uparrow, 0\rangle,|0, \downarrow\rangle)$, the integral over $x_{p}$ is equal to zero. This situation is equivalent to choose an imaginary and temperature-dependent chemical potential in the Popov-Fedotov method [16]. Therefore, the main difference between both models is that in the $2 \mathrm{~S}$ model the contribution of the non-magnetic local states is exactly canceled, while in the $4 \mathrm{~S}$ model it is adopted the half-filling situation. These results are used in Eq. (15) and the free energy can be written as:

$$
\beta F_{s}=\beta^{2} J^{2}\left(q_{a} \bar{\chi}_{b}+q_{b} \bar{\chi}_{a}+\bar{\chi}_{a} \bar{\chi}_{b}\right)-\beta J_{0} m_{a} m_{b}-\sum_{p=a, b} \int_{-\infty}^{\infty} D z_{p} \ln \Theta_{s, p}-\ln 4
$$

with

$$
\Theta_{s, p}=\frac{s-2}{2}+\int_{-\infty}^{\infty} D \xi_{p} \cosh \left(\beta \sqrt{\Delta_{p}}\right)
$$

where $s$ represents the states number allowed in each model. The order parameters $q_{p}, \bar{\chi}_{p}$ and $m_{p}$ are given by extreme condition of the free energy (23):

$$
\begin{aligned}
m_{p} & =\int_{-\infty}^{\infty} D z_{p} \frac{\int_{-\infty}^{\infty} D \xi_{p} h_{p} \sinh \left(\beta \sqrt{\Delta_{p}}\right) / \sqrt{\Delta_{p}}}{\Theta_{s, p}}, \\
q_{p} & =\int_{-\infty}^{\infty} D z_{p}\left(\frac{\int_{-\infty}^{\infty} D \xi_{p} h_{p} \sinh \left(\beta \sqrt{\Delta_{p}}\right) / \sqrt{\Delta_{p}}}{\Theta_{s, p}}\right)^{2}, \\
\bar{\chi}_{p} & =\int_{-\infty}^{\infty} D z_{p} \frac{\int_{-\infty}^{\infty} D \xi_{p}\left[h_{p}^{2} \cosh \left(\beta \sqrt{\Delta_{p}}\right) / \Delta_{p}+\Gamma^{2} \sinh \left(\beta \sqrt{\Delta_{p}}\right) /\left(\beta \Delta_{p}^{3 / 2}\right)\right]}{\Theta_{s, p}}-q_{p} .
\end{aligned}
$$

In particular, when $\Gamma=0$, the set of integrals over $\xi_{p}$ can be performed analytically. In this case, it is easy to see that the parameter $\bar{q}_{p} \equiv \frac{4}{N}\left\langle\sum_{i_{p}} S_{i_{p}}^{\alpha} S_{i_{p}}^{\alpha}\right\rangle=q_{p}+\bar{\chi}_{p}=1$ (Eq. (27)) for the $2 \mathrm{~S}$ model. However, in the $4 \mathrm{~S}$ model, due to the presence of non-magnetic states, $\bar{q}_{p}$ depends on the temperature and the internal field $h_{p}$. Nevertheless, near $T=0, \bar{q}_{p}$ can be expressed by using a low-temperature expansion:

$$
\bar{q}_{p}=1-\frac{(s-2)}{2} \frac{T}{J} \sqrt{\frac{\pi}{4 q_{p^{\prime}}}} \exp \left[-\frac{\left(\varrho_{p}\right)^{2}}{2}-\frac{J \mathrm{e}^{-\left(\varrho_{p}\right)^{2} / 2}}{T \sqrt{q_{p^{\prime}}} \pi}\right]
$$

where $\varrho_{p}=\frac{J_{0} m_{p^{\prime}}}{J \sqrt{2 q_{p^{\prime}}}}$. This result suggests that the occupation of non-magnetic states are exponentially small at low $T$.

It is well known that the replica symmetric solution can be unstable at low temperature in the spin glass phase [5], where the Almeida-Thouless eigenvalue $\lambda_{A T}$ becomes negative. The equation for the $\lambda_{A T}$ following as [8]:

$$
\lambda_{A T}=1-2(\beta J)^{4} \prod_{p=a, b} \int_{-\infty}^{\infty} D z_{p}\left(\frac{I_{p}}{\Theta_{s, p}^{2}}\right)^{2}
$$




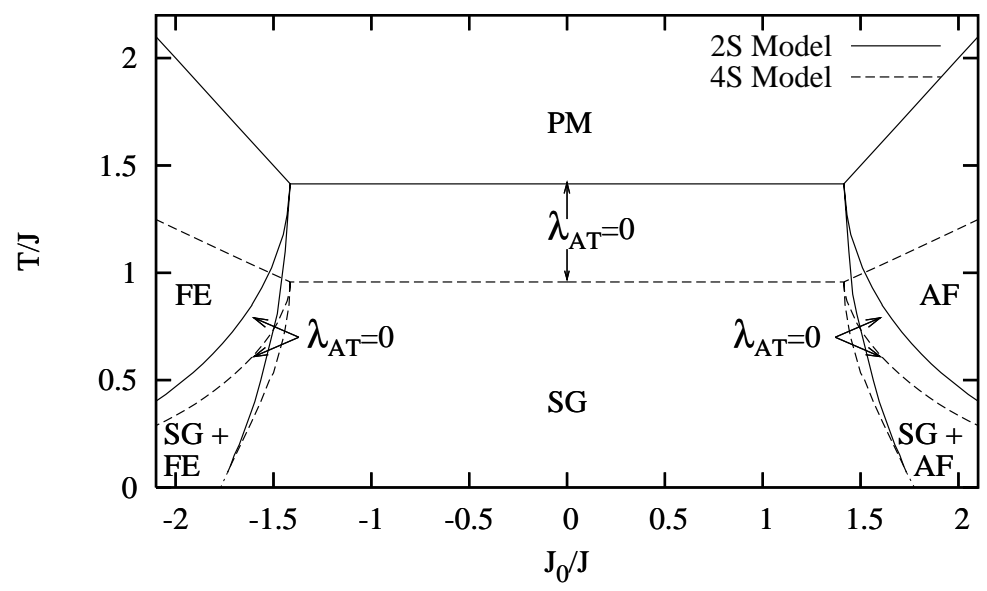

Figure 1: Phase diagram $T / J$ versus $J_{0} / J$ for $H=\Gamma=0$. The solid lines correspond to the results obtained by the restricted model $(2 \mathrm{~S})$, while the dashed lines correspond to the unrestricted model $(4 \mathrm{~S})$. The dotted lines are the extrapolation carried for lower temperature. $\lambda_{A T}=0$ is the AlmeidaThouless line.

where

$$
\begin{aligned}
I_{p}(z)=\Theta_{s, p} \int_{-\infty}^{+\infty} D \xi_{p}\left[\frac{h_{p}^{2}}{\Delta_{p}}\right. & \left.\cosh \left(\beta \sqrt{\Delta_{p}}\right)+\frac{\Gamma^{2}}{\beta \Delta_{p}^{3 / 2}} \sinh \left(\beta \sqrt{\Delta_{p}}\right)\right] \\
& -\left(\int_{-\infty}^{+\infty} D \xi_{p} \frac{h_{p}}{\sqrt{\Delta_{p}}} \sinh \left(\beta \sqrt{\Delta_{p}}\right)\right)^{2} .
\end{aligned}
$$

\section{Results}

The phase diagrams showing the competition between SG and AF for both models with two (2S) and four $(4 \mathrm{~S})$ states can be obtained solving the replica symmetric order parameters given by the set of equations (25)-(27). In the present work, the numerical solutions for $m_{p}, q_{p}, \bar{\chi}_{p}$ and $\lambda_{A T}$ (with $p=a$ or $b$ ) are studied by varying the three relevant parameters in the problem, which are $J_{0} / J, \Gamma / J$ and $H / J$. In this context, the AF phase is given by the order parameters $l_{m}=m_{a}-m_{b} \neq 0$ and $q_{a}-q_{b} \neq 0$; however, the same situation with the eigenvalue $\lambda_{A T}<0$ characterizes the mixed phase $(\mathrm{SG}+\mathrm{AF})$. It can be considered that, in the $\mathrm{SG}$ phase, the order parameters $l_{m}=0$ and $q_{a}=q_{b} \neq 0$ together with $\lambda_{A T}<0$. In this quantum fermionic treatment, one can identify two situations for the diagonal component of the replica matrix $\left(\bar{q}_{p}=q_{p}+\bar{\chi}_{p}\right)$. For the $2 \mathrm{~S}$ model with $\Gamma=0, \bar{q}_{p}=1$. On the other hand, for the $4 \mathrm{~S}$ model or for the $2 \mathrm{~S}$ model when $\Gamma>0$, it is necessary to consider the coupling between diagonal and off-diagonal replica matrix elements. Consequently, the parameter $\bar{\chi}_{p}$ must be calculated simultaneously with $q_{p}$ and $m_{p}$, and hence it becomes relevant for determination of the remaining physical quantities. In particular, for $H=0$, the Neel temperature $T_{N 0}=T_{N}\left(J_{0}, \Gamma, H=0\right)$ can be computed expanding the saddle point equations in powers of $m_{p}$. At the second order critical line $T_{N 0}$, we can make $q_{p}=0$, therefore $\bar{\chi}=\bar{\chi}_{p}=1 /\left(\beta_{c} J_{0}\right)$ and

$$
m\left[1-\beta_{c} J_{0}\left(\frac{\frac{s-2}{2}+\int D \xi \xi^{2} \cosh \beta_{c} \sqrt{\frac{2 J \xi^{2}}{J_{0} \beta_{c}}+\Gamma^{2}}}{\frac{s-2}{2}+\int D \xi \cosh \beta_{c} \sqrt{\frac{2 J \xi^{2}}{J_{0} \beta_{c}}+\Gamma^{2}}}\right)\right]=0,
$$




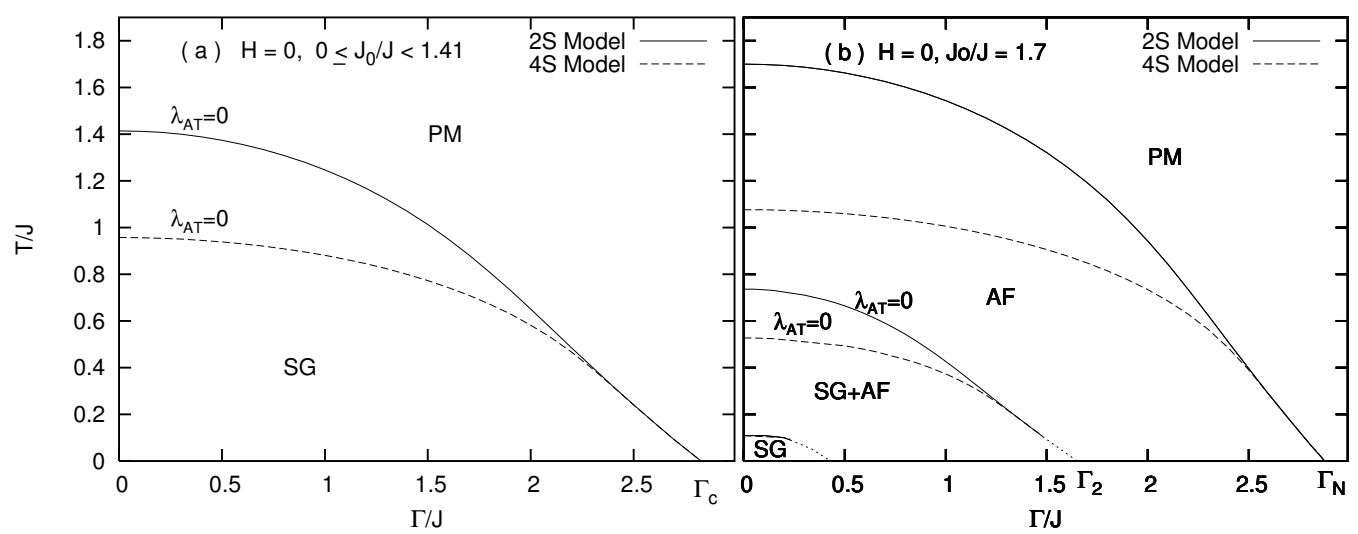

Figure 2: Phase diagrams $T / J$ versus $\Gamma / J$ for $H=0$, (a) $0 \leq J_{0} / J<\sqrt{2}(\alpha>1 / \sqrt{2})$ and (b) $J_{0} / J=1.7(\alpha=0.59)$. The line conventions are the same as in Fig. (1).

where $\beta_{c}=1 / T_{N 0}$ and $m=m_{p}=-m_{p^{\prime}}$. For $\Gamma=0$, the Neel temperature is $T_{N 0}=J_{0} /\left[\frac{s-2}{2}\right.$ $\left.\exp \left(\frac{-J^{2}}{T_{N 0} J_{0}}\right)+1\right]$, while for $T_{N 0}$ close to zero, the critical value of the transverse field is given by analytical solution of Eq. (31): $\Gamma_{N}=J_{0}+2 J^{2} / J_{0}$, for both models.

In Fig. (1), it is shown the diagram $T / J$ ( $T$ is the temperature) versus $J_{0} / J$ when $\Gamma$ and $H$ are zero. For that particular situation, the results for $2 \mathrm{~S}$ and $4 \mathrm{~S}$ models are qualitative equivalent to the KS model with classical Ising spins. Actually, for the $2 \mathrm{~S}$ model, the Fischer relation [3] is recovered $\bar{\chi}_{a, b} \equiv \chi_{a, b}^{*} / \beta=1-q_{a, b}$ ( $\chi_{a, b}^{*}$ is the linear susceptibility for $J_{0}$ equal to zero). Therefore, the $2 \mathrm{~S}$ model reproduces exactly the KS results 8 . For high temperature and high degree of frustration $\alpha \equiv\left[J_{0} / J\right]^{-1}$, a paramagnetic phase (PM) is found. The AF solution for both models can be found in the region where the degree of frustration $\alpha$ is small enough. In this case, $m_{a}=-m_{b}$ [8]. For increasing $\alpha$ and decreasing temperature, the Almeida-Thouless line in Fig. (1) shows the onset (at freezing temperature $T_{f}$ ) of the complex ergodicity breaking region (the $\mathrm{SG}$ region). There is also an intermediate mixed phase region ( $\mathrm{SG}+\mathrm{AF})$, where inside the $\mathrm{SG}$, the magnetization of the sublattices remains finite with opposite sign. It should be remarked that the transition temperatures are different in both models. Particularly, for the same $\alpha,\left(T_{f}\right)_{2 S}>\left(T_{f}\right)_{4 S}$, $\left(T_{N}\right)_{2 S}>\left(T_{N}\right)_{4 S}$ and $\left(d T_{N} / d \alpha\right)_{2 S}<\left(d T_{N} / d \alpha\right)_{4 S}\left(T_{N}\right.$ is the Neel temperature). These distinct behaviors show the different sensitivity to the magnetic coupling between the two models. For the sake of completeness, in Fig. (1), it is also studied the region for $J_{0} / J<0$ where a ferromagnetic (FE) region is found with $m_{a}=m_{b}$. Thus, one recovers essentially the one lattice spin glassferromagnetism competition [8, 17. These results show that, in absence of magnetic fields, there is a symmetry between the two sublattices.

If $\Gamma$ is turned on (with $H=0$ ), two distict situations can be identified. For the degree of frustration $\alpha>1 / \sqrt{2}\left(0 \leq J_{0} / J<\sqrt{2}\right)$, the magnetization of both sublattices is zero. Therefore, the problem is reduced to the one lattice problem in the presence of a transverse field $\Gamma$ studied in reference [5] (see Fig. (2-a)). For small $\Gamma$, the freezing temperature $T_{f}$ is different for each model. However, when the temperature is decreased and the spin flipping increases due to $\Gamma$, the critical behavior of both models tends to become identical. The critical field $\Gamma_{c}$, at the QCP for $2 \mathrm{~S}$ and $4 \mathrm{~S}$ models, is the same. The Fig. (2-b) shows a phase diagram for a smaller degree of frustration $\alpha=0.59\left(J_{0} / J=1.7\right)$. For this set of parameters, the PM solution is still found at high temperature for any value of $\Gamma$. For small values of $\Gamma$, when the temperature is decreased, there is a transition to the AF phase at $\left(T_{N}\right)_{2 S, 4 S}$, but $\left(T_{N}\right)_{2 S}>\left(T_{N}\right)_{4 S}$. For even lower temperature, there is other transition to the mixed phase (SG $+\mathrm{AF}$ ) at $\left(T_{f}\right)_{2 S, 4 S}$ (again $\left(T_{f}\right)_{2 S}>\left(T_{f}\right)_{4 S}$ ), and finally to the SG phase. These transitions are mainly thermally driven. On the other hand, when $\Gamma$ is increased, the Neel temperature $T_{N}$ for both models decreases towards a QCP at $\Gamma_{N}$. The temperatures $\left(T_{f}\right)_{2 S, 4 S}$, given by the AT line, decrease when $\Gamma$ increases like the Neel temperature. 


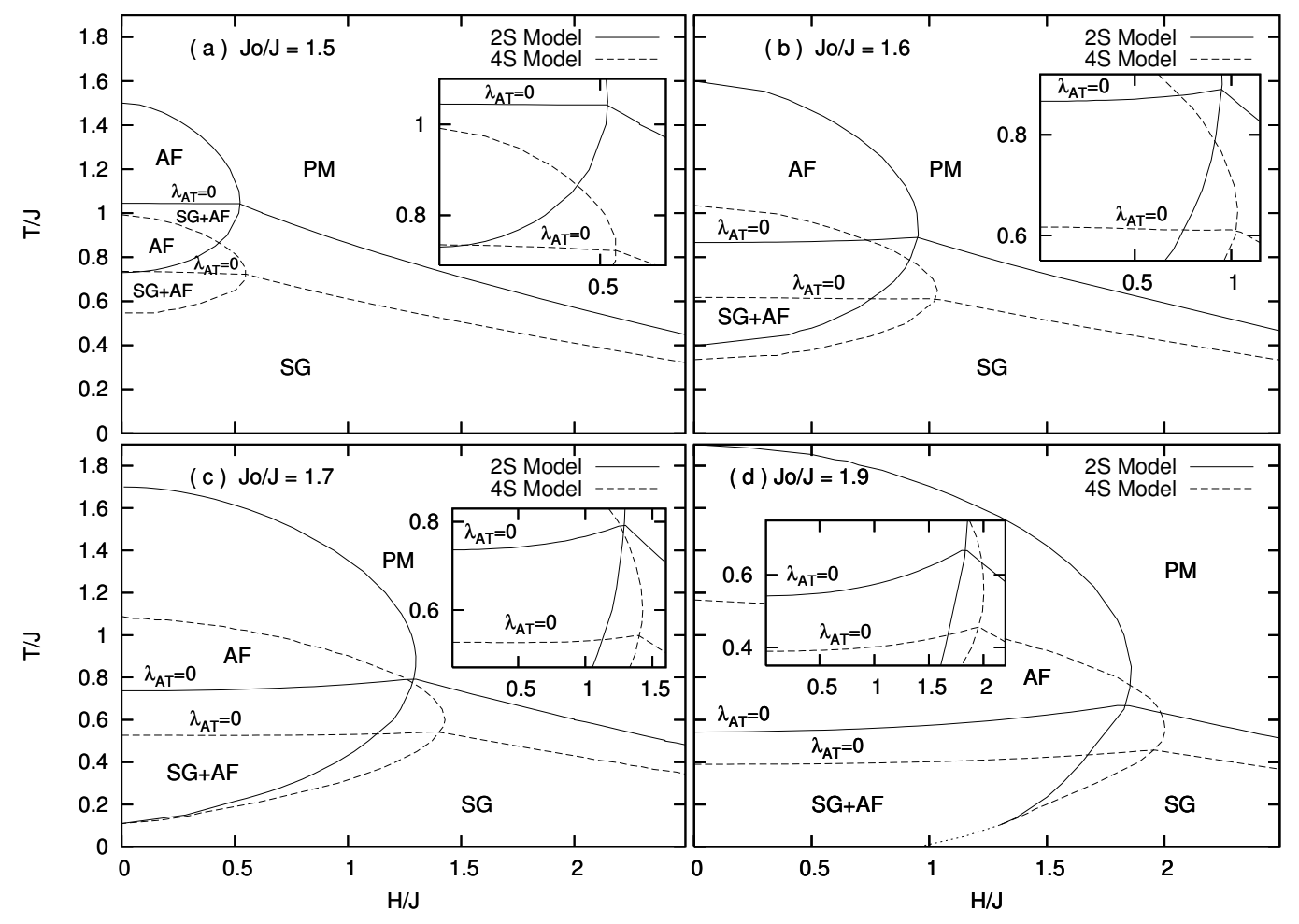

Figure 3: Phase diagrams $T / J$ versus $H / J$ for $\Gamma=0$ and several values of $J_{0} / J$ : (a) $J_{0} / J=1.5$ $(\alpha=0.67)$, (b) $J_{0} / J=1.6(\alpha=0.63)$, (c) $J_{0} / J=1.7(\alpha=0.59)$, and (d) $J_{0} / J=1.9(\alpha=0.53)$. The insets represent a zoom in $T$ near $T_{f}$. It is used the same convention as Fig. (1) for the line types.

We can observe that both models converge to the same critical behavior when $\Gamma$ is increased. The reasons for that behavior at $\alpha=0.59$ are the same for large degree of frustration $(\alpha>1 / \sqrt{2})$. When we see Eq. (24), at very low temperature, for the $4 \mathrm{~S}$ model, the term which is integrated over $\xi_{p}$ is dominant and the distinction between the two models can be neglected. It occurs because the contribution of magnetic states dominate the free energy [5]. Therefore, there is a distinction between the models in the region where the transitions are mostly thermally driven.

Fig. (3) shows the phase diagrams $T / J$ versus $H / J$ when $\Gamma=0$. These phase diagrams are studied with four different values of degree of frustration $\alpha$. The field $H$ breaks the symmetry between the sublattices and produces an effect similar to the KS model [8, 18. It tends to destroy the AF phase. For instance, the Neel temperatures $\left(T_{N}\right)_{2 S, 4 S}$ decrease fast when $H$ is enhanced in the region $0<H<H_{c}\left(H_{c}\right.$ is the magnetic field when $\left.\left(T_{f}\right)_{s}=\left(T_{N}\right)_{s}(s=2 S, 4 S)\right)$. However, as one can see quite clearly in Figs. (3-b)-(3-d), there is a range of magnetic field close to $H_{c}$ where the freezing temperatures, associated with the AT instability, increase showing that the frustration is favored in this two-sublattice problem 8,18 . This behavior is different from the one lattice problem where the $T_{f}$ decreases monotonically [9] for any value of $H$. Nevertheless, for $H>H_{c}$, the behavior of the $\mathrm{AT}$ line for $2 \mathrm{~S}$ and $4 \mathrm{~S}$ models becomes similar to the one lattice problem. The favoring of frustration for both models as function of $H$ can be related to drastic decreasing of Neel temperature [18 near $H_{c}$. Despite this, the presence of the magnetic field produces different effects for $2 \mathrm{~S}$ and $4 \mathrm{~S}$ models. The freezing temperature, given by the AT instability, increases faster in the $2 \mathrm{~S}$ model than in the $4 \mathrm{~S}$ for increasing $H$. The $\mathrm{AF}$ solution is also more robust for the $4 \mathrm{~S}$ model, it is found for greater values of $H$ than the $2 \mathrm{~S}$ model.

In Fig. (4), the phase diagrams $T / J$ versus $H / J$ is shown, but with the transverse field tunned $(\Gamma / J=1.0)$. The set of values of $\alpha$ is the same as Fig. (3). For that situation, there are very 


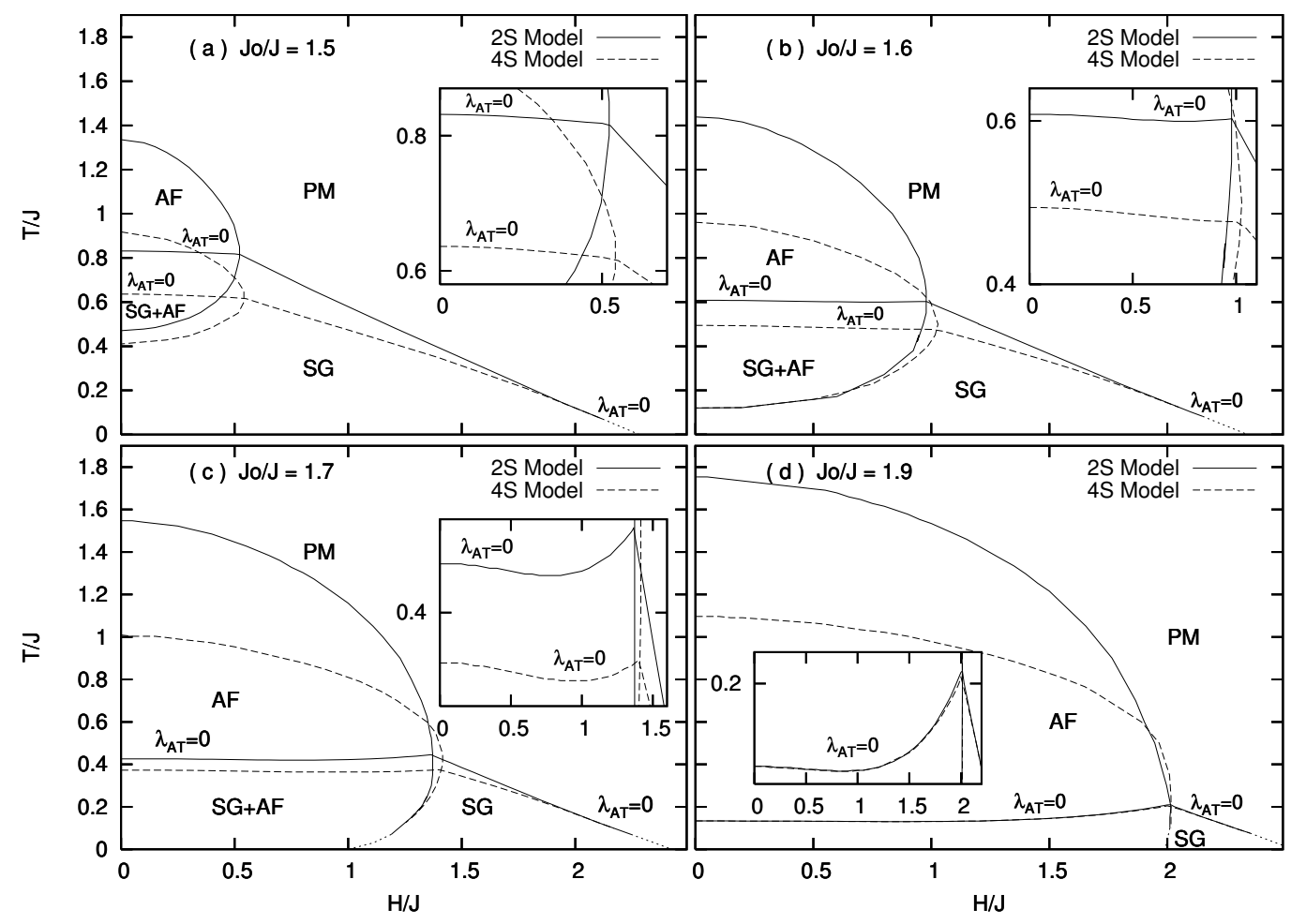

Figure 4: Phase diagrams $T / J$ versus $H / J$ for $\Gamma / J=1.0$ and several values of $J_{0} / J$ : (a) $J_{0} / J=1.5$ $(\alpha=0.67)$, (b) $J_{0} / J=1.6(\alpha=0.63)$, (c) $J_{0} / J=1.7(\alpha=0.59)$ and (d) $J_{0} / J=1.9(\alpha=0.53)$. The same convention as Fig. (3) is used for the line types and the insets. 


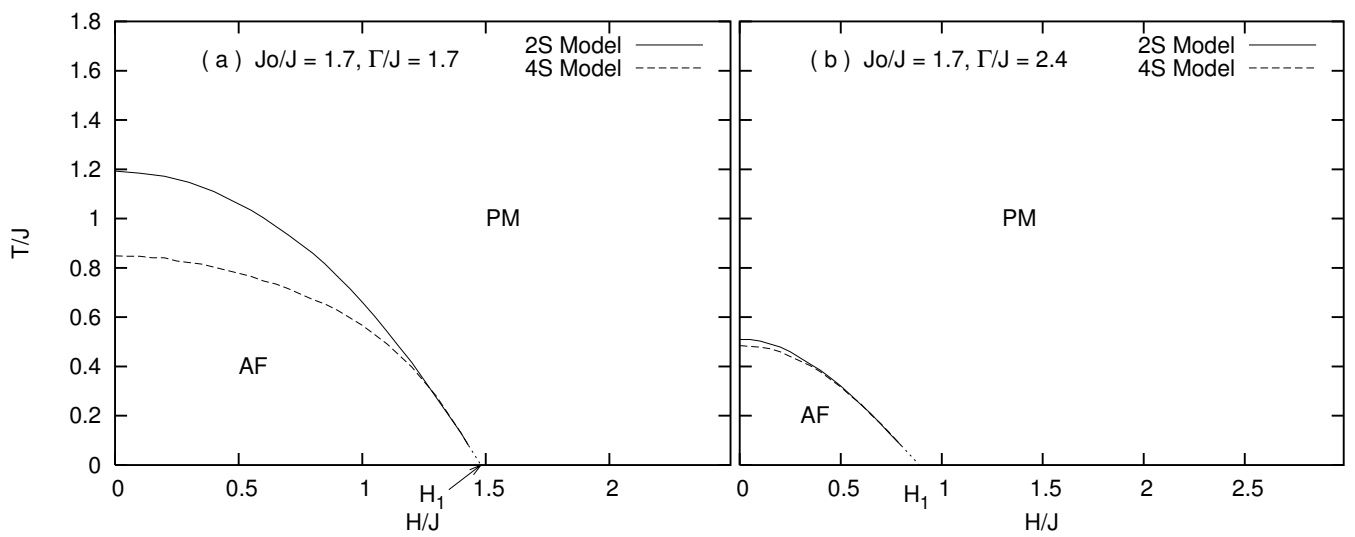

Figure 5: Phase diagrams $T / J$ versus $H / J$ for $J_{0} / J=1.7(\alpha=0.59)$ and: (a) $\Gamma / J=1.7$, (b) $\Gamma / J=2.4$. The same convention as Fig. (1) is used for line types.

important differences if compared with the $\Gamma=0$ situation. One effect of the applied $\Gamma$ is to depress $\left(T_{N}\right)_{2 S, 4 S}$ and $\left(T_{f}\right)_{2 S, 4 S}$ for any value of $H$. Other important effect is that the AT lines for both models have a quite different behavior in the range $0<H<H_{c}$. For instance, in Fig. (4-a) where $\alpha=0.67\left(J_{0} / J=1.5\right)$, when $H$ is enhanced until $H_{c}$, the $T_{f}$, associated with the AT instability, decreases faster than the equivalent phase diagram with $\Gamma=0$ (see Fig. (3-a)). When $\alpha$ is increased (see Figs. (4-b)-(4-c)), the AT line shows the delicate balance between the mechanisms enforced by $\Gamma$ and $H$. For $H \approx 0$, where the presence of $\Gamma$ is dominant, the spin flipping tends to destroy frustration $\left(d T_{f} / d H<0\right)$. As long as $H$ increases, the frustration is favored again. When $H \approx H_{c}$, it is quite clear that $d T_{f} / d H>0$. Particularly, close to $H_{c}$, there is a strong increase in the $T_{f}$ as $\alpha$ decreases. When the $\Gamma$ field is enhanced as in Fig. (5) (where $\Gamma / J=1.7$ and $\Gamma / J=2.4$ with the degree of frustration $\alpha=0.59$ ), the SG character is entirely suppressed. The Neel temperature decreases towards $H_{1}$. The differences between $2 \mathrm{~S}$ and $4 \mathrm{~S}$ models still exist for $H \approx 0$. However, the increase of $\Gamma$ shifts the Neel temperature of both models towards zero. As consequence, the difference $\left(T_{N}\right)_{2 S}-\left(T_{N}\right)_{4 S}$ is increasily small.

Finally, the study of the magnetic susceptibility allows one to confirm the position of the Neel temperatures and to study in detail the differences between the $2 \mathrm{~S}$ and $4 \mathrm{~S}$ models near $T_{N}$. The magnetic susceptibility $\chi$ is obtained differentiating the saddle point equations (25] 26. 27) with respect to $\mathrm{H}$. In the limit of zero field $(H \rightarrow 0)$, the six-independent saddle point equations system is reduced to a three-independent saddle point equations with the following relations: $m_{a}(H=0)=-m_{b}(H=0), q_{a}(H=0)=q_{b}(H=0)$ and $\bar{\chi} \equiv \bar{\chi}_{a}(H=0)=\bar{\chi}_{b}(H=0)$. In this case, the resulting linear system for $\partial m_{p} / \partial H, \partial q_{p} / \partial H$ and $\partial \bar{\chi}_{p} / \partial H(p=a$ or $b)$ is also simplified giving $\chi=\partial m_{a} / \partial H=\partial m_{b} / \partial H, \partial q_{a} / \partial H=-\partial q_{b} / \partial H, \partial \bar{\chi}_{a} / \partial H=-\partial \bar{\chi}_{b} / \partial H$ [8]. Thus,

$$
\chi=\frac{\bar{\chi}+\Pi}{T+J_{o}(\bar{\chi}+\Pi)}
$$

where $\bar{\chi}$ is given in Eq. (27) and $\Pi$ is defined in Eq. (34) of the Appendix.

For high temperature, in the PM phase, the susceptibility has the expression $\chi=\bar{\chi} /\left(T+J_{0} \bar{\chi}\right)$, as expected. Now, when the temperature is decreased, $\chi$ shows a brusque change in its inclination at $T_{N}$ and a cusp at $T_{g}\left(T_{g}\right.$ is the temperature below $T_{f}$, where $\left.m_{p}=m_{p^{\prime}}=0\right)$, but it does not show any anomaly at $T_{f}$, as we can see in Fig. (6). For intermediaries values of $J_{0}$, the susceptibility in the AF phase near $T_{N}$ does not decrease with the decrease of temperature (inset of Fig. (66-a)). However, it exhibits a slow increase until reaching a maximum value, then it decreases. That is because of the large number of frustrated couplings. Nevertheless, strong antiferromagnetic average coupling, for example $J_{0} / J=2.5, \chi$ has a maximum in $T_{N}$ for both models (Fig. 66 c)). In Fig. ([6]-b), for $J_{0} / J=2.1$ and $\Gamma=H=0, \chi$ exhibits a maximum at $T_{N}$ only for the $2 \mathrm{~S}$ model, while 

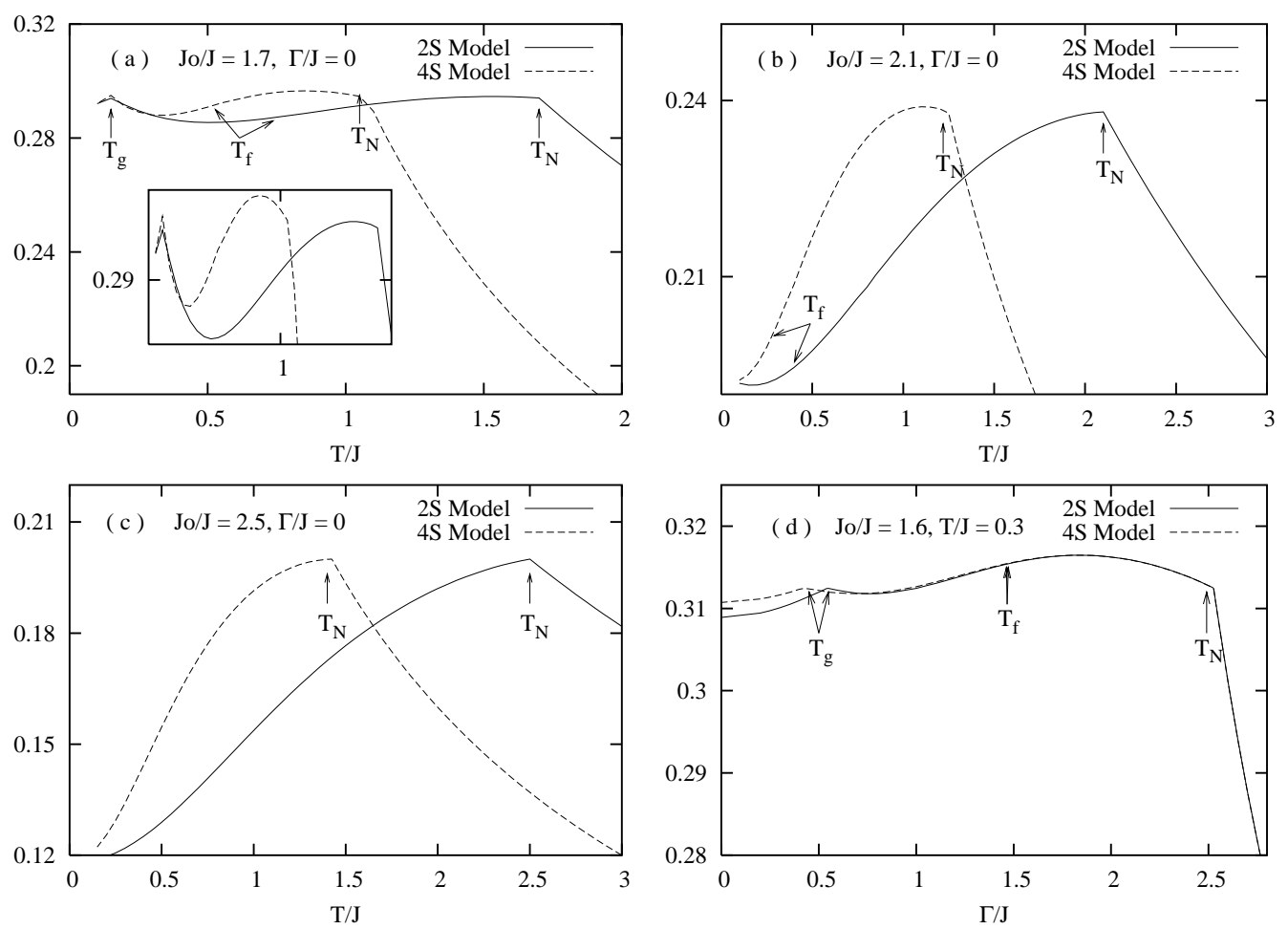

Figure 6: The figures (a), (b) and (c) are plots of $\chi$ versus $T / J$ for $\Gamma=H=0$ and several values of $J_{0}(1.7 ; 2.1 ; 2.5)$. The figure $(\mathrm{d})$ is the $\chi$ as a function of $\Gamma / J$ for $H=0, T=0.3 J$ and $J_{0}=1.6 J$. It is used the same convention as Fig. (1) for the line types. The inset in (a) represents a zoom of $\chi$ near the critical points.

for the $4 \mathrm{~S}$ model, $\chi$ still increases close to $T_{N}$ in the $\mathrm{AF}$ phase. Therefore, due to the presence of non-magnetic states in the $4 \mathrm{~S}$ model, its frustrated couplings are less sensitive than the $2 \mathrm{~S}$ model ones for the increasing of $J_{0}$. Another difference of the models is in the value of the critical points at high temperatures as showed in Fig. (6). The Fig. (6-d) shows the $\chi$ for $T=0.3 J, H=0$, and $\alpha=.625$ as a function of $\Gamma$. Again, the critical points are marked by discontinuities in the $\chi$ that is obtained changing the transverse field. These discontinuities have the same shape described above.

\section{Conclusion}

In the present work, the competition between antiferromagnetism (AF) and spin glass (SG) in disordered fermionic Ising models has been studied. The $J_{i j}$ coupling among the spins is a random gaussian variable with average and variance given by $-4 J_{0} / N$ and $32 J^{2} / N$, respectively. There is also a magnetic field applied with components transverse $(\Gamma)$ and parallel $(H)$ to the Ising spin direction. The model is similar to the Korenblit-Shender model (KS) 8 for classical Ising spin which consists of two sublattices where only the interlattice frustration has been considered.

The problem has been formulated in the path integral formalism where the spin operators are represented by bilinear combinations of Grassmann fields following closely the approach introduced in a previous work [5, which has studied the SG critical properties in presence of a transverse field using the static approximation. The focus in this work has been to investigate the boundary phase of the SG/AF competition in this two-sublattice model 8 for Ising spins in its fermionic representation. For that purpose, the static approximation has been adopted like the previous work in Ref. [5]. Two formulations for the fermionic representation for the operator $S_{i}^{z}$ have 
been assumed: the first one is the unrestricted four-state 4S model (four eingenvalues, two of them magnetically insensitive); the second one is the restrict $2 \mathrm{~S}$ model where the non-magnetic eigenvalues are forbidden by an imposed constraint. It should be remarked that the transverse component $\Gamma$ can tune spin flipping while $H$ destroys the symmetry between the sublattices. Therefore, these components have opposite roles as related to the emergence of frustration in the problem.

If $\Gamma=0$, the Fischer [19] relation $\bar{\chi}_{p}=1-q_{p}(p=a, b)$ is recovered in the $2 \mathrm{~S}$ model, as we can see from the equations (25)-(27). Thus, the results of the KS model are replicated [8]. That is an important difference with the $4 \mathrm{~S}$ model, where the Fischer relation is not satisfied due to the coupling between diagonal and off-diagonal replica matrix elements. Therefore, the internal field $h_{p}$ (see Eq. 21) behaves in a quite distinct way for both models. These differences can be seen very clearly in the phase diagram temperature versus $J_{0} / J$ given in Fig. (1) with $H=0$. The onset of AF (at $T_{N}$ ) and the complex ergodicity breaking given by the Almeida-Thouless (AT) line (at $T_{f}$ ) appear for lower temperatures in the $4 \mathrm{~S}$ model compared with the $2 \mathrm{~S}$ one. That is a direct consequence of the distinct applied internal fields for each model. In the susceptibility $\chi$, given in Eq. (32), these differences can be seen even in a more detailed way (see figures (6-a)-(6-c)).

When the transverse component $\Gamma$ is turned on ( $H$ is kept equal to zero) (see Fig. (2)), there is an important change in the critical behavior for both models. In the range of degree of frustration $\alpha \equiv\left[J_{0} / J\right]^{-1}>1 / \sqrt{2}\left(0 \leq J_{0} / J<\sqrt{2}\right)$, the problem is reduced to the one lattice problem studied in Ref. [5]. For small $\Gamma$, there are two different transition temperatures from paramagnetism $(\mathrm{PM})$ to $\mathrm{SG}$ for each model. These transition temperatures converge to same value as $\Gamma$ increases given origin to the same $\mathrm{QCP}$ at $\Gamma_{c}$. For $\alpha<1 / \sqrt{2}$, for small $\Gamma$, when the temperature is lowered, there are the following sequences of transitions: (1) PM/AF at $T_{N}\left(\left(T_{N}\right)_{2 S} \neq\left(T_{N}\right)_{4 S}\right) ;(2)$ $\mathrm{AF} /(\mathrm{AF}+\mathrm{SG})$, given by the position of the Almeida-Thouless $(\mathrm{AT})$ line at $T_{f}\left(\left(T_{f}\right)_{2 S} \neq\left(T_{f}\right)_{4 S}\right)$, where $(\mathrm{AF}+\mathrm{SG})$ is a mixed phase; $(3)(\mathrm{AF}+\mathrm{SG}) / \mathrm{SG}$ at $T_{g}\left(\left(T_{g}\right)_{2 S} \neq\left(T_{g}\right)_{4 S}\right)$, where at this transition temperature the sublattice magnetizations become null. For increasing $\Gamma$, the $T_{N}$ for each model decreases converging to the same transition line. The same effect is found for $T_{f}$. A QCP for PM/AF transition is found at $\Gamma_{N} / J=J_{0} / J+2 J / J_{0}$.

Other important consequence appears when the parallel field $H$ is turned on and, consequently, the symmetry between the sublattices is broken. That is source of unusual effects because the internal field applied in the sublattice $p=a, b$ depends on the order parameters of the other sublattice $p^{\prime}=b, a[8]$ [18. For the $2 \mathrm{~S}$ model, the results of the KS model are reproduced [8] (see Fig. (3)). The Neel temperatures for both models (which are different) have a strong decrease as $H$ increases. However, the frustration is favored for a particular range of $H$ as shown in Fig. (3) from the AT line behavior. It occurs due to the magnetization of a particular sublattice (for instance $p$ where $m_{p}>m_{p^{\prime}}$ ) which contributes to decrease the absolute value of the average internal field $\left(\bar{h}_{p}=H-J_{0} m_{p^{\prime}}\right)$ that acts on the other one. Hence, in this $H$ range where $\bar{h}_{p}$ decreases with $H$, $T_{f}$ can be increased [8]. That effect is stronger when $\alpha$ is decreased $\left(J_{0} / J\right.$ is enhanced), since the contribution of $J_{0} m_{p^{\prime}}$ on $\bar{h}_{p}$ is increased, as shown in Figs. (3-b)-(3-c). Because of the presence of non-magnetic state, the $4 \mathrm{~S}$ model has potentially a weaker response to the magnetic interactions. The results show that the AT line can also increase, but slower than the $2 \mathrm{~S}$ model for the same range of $H$. Nevertheless, in the $4 \mathrm{~S}$ model, the AF phase is more robust than the $2 \mathrm{~S}$ one, remaining as solution even for higher values of $H$. However, for very low temperature, the contribution of the non-magnetic states in the $4 \mathrm{~S}$ model becomes less significant. Therefore, both models exhibit quite similar critical lines.

When the transverse field $\Gamma$ and $H$ are simultaneously turned on, the effective internal field applied in a particular sublattice $p$ becomes $\Delta_{p}=\sqrt{h_{p}^{2}+\Gamma^{2}}$. Therefore, its effects due to the presence of $H$ and $\Gamma$ start to compete. The effects from $H$ in the $h_{p}$ are included explicitly, but they are also included implicitly by the order parameters. It should be noticed that effects of $\Gamma$ in the order parameters are present as well. As consequence, there is complex balance of the effects discussed in the previous paragraph, dependent on the relation $H / \Gamma$. Particularly, for small $H$, the contribution from $\Gamma$ is dominant and the frustration is not favored. As long as $H$ enhances, there is a region in the diagram temperature versus $H$ where the frustration becomes favored (see 
Fig. (4)). Therefore, the transverse component of the magnetic field produces the same effect in each sublattice. It suppresses the magnetic order and the frustration. While, in the region where a two-sublattice structure is characterized, the parallel component acts in an asymmetric way in each sublattice. In this sense, it is always against the AF order. Nevertheless, it can favor frustration within a certain range of $H$.

To conclude, we studied the SG/AF competition in a two sublattice model [8] where the Ising spins have a fermionic representation. There is a magnetic field applied. This field has a transverse component $(\Gamma)$ which can flip the spins leading the transitions to a QCP. It has also a parallel component $(H)$ which breaks the symmetry between the sublattices. These components produce opposite effects on the frustration. Our hope is to use this representation to study the several strongly correlated problems where there is a $\mathrm{SG} / \mathrm{AF}$ competition which is associated with a QCP as, for instance, in the Cerium alloy $C e_{2} A u_{1-x} C o_{x} S i_{3}$ [13. On the other hand, we have used the static approximation and replica symmetry ansatz. Quite recently, a new scheme for breaking replica symmetry has been proposed [20] which is particularly suitable for the present two-sublattice problem studied here. That could be used to improve considerably the description of the SG/AF competition of our fermionic model. That would be an object for future work.

\section{Acknowledgments}

The numerical calculations were, in part, performed at LSC (Curso de Ciência da Computação, UFSM) and at Grupo de Física Estatítica-IFM, Universidade Federal de Pelotas. This work was partially supported by the Brazilian agency CNPq (Conselho Nacional de Desenvolvimento Científico e Tecnológico). The authors are grateful to Prof. Alba Theumann for useful discussions.

\section{A Appendix}

In this appendix, the function $\Pi$ (see Eq. (32) ) is found explicitly:

$$
\begin{aligned}
\Pi & =\frac{\beta^{2} J^{2} K_{2}\left(F_{2}-F_{3}\right)}{1+\beta^{2} J^{2} K_{1}}-\frac{\beta^{2} J^{2} F_{3}}{1+\beta^{2} J^{2} F_{4}}\left(F_{3}-\frac{K_{2}\left(1+\beta^{2} J^{2} F_{6}\right)}{1+\beta^{2} J^{2} K_{1}}\right), \\
K_{1} & =F_{1}-\frac{2 F_{5}\left(1+\beta^{2} J^{2} K_{2} F_{6}\right)}{1+\beta^{2} J^{2} F_{4}}, \\
K_{2} & =F_{2}-\frac{2 \beta^{2} J^{2} K_{2} F_{3} F_{5}}{1+\beta^{2} J^{2} F_{4}}
\end{aligned}
$$

where:

$$
\begin{aligned}
& F_{1}=\int_{-\infty}^{\infty} D z \frac{\partial^{2}}{\partial h^{2}}\left[\frac{\int_{-\infty}^{\infty} D \xi \partial \cosh \sqrt{\Delta} / \partial h}{s+\int_{-\infty}^{\infty} D \xi \cosh \sqrt{\Delta}}\right]^{2}, \\
& F_{2}=\int_{-\infty}^{\infty} D z \frac{\partial}{\partial h}\left[\frac{\int_{-\infty}^{\infty} D \xi \partial \cosh \sqrt{\Delta} / \partial h}{s+\int_{-\infty}^{\infty} D \xi \cosh \sqrt{\Delta}}\right]^{2}, \\
& F_{3}=\int_{-\infty}^{\infty} D z \frac{\partial}{\partial h}\left[\frac{\int_{-\infty}^{\infty} D \xi \partial^{2} \cosh \sqrt{\Delta} / \partial h^{2}}{s+\int_{-\infty}^{\infty} D \xi \cosh \sqrt{\Delta}}\right], \\
& F_{4}=\int_{-\infty}^{\infty} D z\left[\frac{\int_{-\infty}^{\infty} D \xi \partial^{4} \cosh \sqrt{\Delta} / \partial h^{4}}{s+\int_{-\infty}^{\infty} D \xi \cosh \sqrt{\Delta}}-\left(\frac{\int_{-\infty}^{\infty} D \xi \partial^{2} \cosh \sqrt{\Delta} / \partial h^{2}}{s+\int_{-\infty}^{\infty} D \xi \cosh \sqrt{\Delta}}\right)\right] \\
& F_{5}=\int_{-\infty}^{\infty} D z\left[\frac{\int_{-\infty}^{\infty} D \xi \partial \cosh \sqrt{\Delta} / \partial h}{s+\int_{-\infty}^{\infty} D \xi \cosh \sqrt{\Delta}} \frac{\partial}{\partial h}\left(\frac{\int_{-\infty}^{\infty} D \xi \partial^{2} \cosh \sqrt{\Delta} / \partial h^{2}}{s+\int_{-\infty}^{\infty} D \xi \cosh \sqrt{\Delta}}\right)\right] \\
& F_{6}=\int_{-\infty}^{\infty} D z \frac{\partial^{2}}{\partial h^{2}}\left[\frac{\int_{-\infty}^{\infty} D \xi \partial^{2} \cosh \sqrt{\Delta} / \partial h^{2}}{s+\int_{-\infty}^{\infty} D \xi \cosh \sqrt{\Delta}}\right],
\end{aligned}
$$

with $\Delta=h^{2}+\beta^{2} \Gamma^{2}$ and $h \equiv \beta h_{a}=\beta h_{b}\left(h_{a}\right.$ is defined in Eq. (21) $)$. 


\section{References}

[1] R. P. Dickey, E. J. Freeman, V. S. Zapf, P. C. Ho, M. B. Maple, Phys. Rev. B 68 (2003) 144402 .

[2] D. J. Scalapino, Phys. Rep. 250 (1995) 329.

[3] Do-Hyun Kim and Jong-Jean Kim, Phys. Rev. B 66 (2002) 054432.

[4] D. Thirumalai, Q. Li, and T. R. Kirkpatrick, J. Phys. A 22 (1989) 3339; P. Ray, B. K. Chakrabarti, and A. Chakrabarti, Phys. Rev. B 39 (1989) 11828; G. Buttner and K. D. Usadel, Phys. Rev. B 41 (1990) 428; Y. Y. Goldschmidt and P. Y. Lai, Phys. Rev. Lett 64 (1990) 2467.

[5] Alba Theumann, A. A. Schmidt, and S. G. Magalhães, Physica A 311 (2002) 498.

[6] W. Wiethege, D. Sherrington, J. Phys. C: Solid State Phys. 19 (1986) 6893; R. Oppermann, A. Muller-Groellig, Nuclear Phys. B 401 (1993) 507; O. Veits, R. Oppermann, M. Bindenberger, J. Stein, J. Phys. I France 4 (1994) 493; V. N Popov, S. A. Fedotov, Sov. Phys. JETP 67 (1988) 535.

[7] Alba Theumann, B. Coqblin, Phys. Rev. B 69 (2004) 214418.

[8] I. Ya. Korenblit and E. F. Shender, Sov. Phys. JETP 62 (1985) 1030.

[9] D. Sherrington and S. Kirkpatrick, Phys. Rev Lett 35 (1975) 1792; S. Kirkpatrick and S. Sherrington, Phys. Rev. B 17 (1978) 4384.

[10] A.J. Bray and M.A. Moore, J. Phys. C13, (1980) L655.

[11] S. G. Magalhães, A. A. Schmidt, F. M. Zimmer, A. Theumann, and B. Coqblin, Eur. Phys. J. B $34(2003) 447$.

[12] S. G. Magalhães and F. M. Zimmer, Eur. Phys. J. B 43 (2005) 187.

[13] S. Majumdar, E.V. Sampathkumaram, St. Berger, M. Della Mea, H. Michor, E. Bauer, M. Brando, J. Hemberger, A. Loidl, Solid State Commun. 121 (2002) 665.

[14] Alba Theumann and M. Vieira Gusmão, Phys. Let. 105A (1984) 311.

[15] J. W. Negele and H. Orland, Quantum Many-Particle Systems (Addison-Wesley Publishing Company, 1988).

[16] V.N. Popov, S.A. Fedotov, Zh. Eksp. Teor. Fiz. 94 (1988) 183.

[17] S. G. Magalhães, A. A. Schmidt, Alba Theumann, B. Coqblin, Eur. Phys. J. B 30 (2002) 419.

[18] I. Ya. Korenblit, Yu. A. Fedorov, E. F. Shender, Sov. Phys. JETP 65 (1987) 400.

[19] K. H. Fischer, Phys. Rev. Lett. 34 (1975) 1438.

[20] R. Oppermann, D. Sherrington, and M. Kiselev, Eur. Phys. J. B 37 (2004) 187. 